\title{
FGF23-Related Hypophosphataemic Bone Disease
}

\author{
Antonio González-Meneses López
}

Received: July 11, 2019 / Published online: March 31, 2020

(C) Springer Healthcare Ltd., part of Springer Nature 2020 symposium sponsored by Kyowa Kirin Pharmaceuticals took place in Madrid, in which national and international experts addressed several aspects of these rare kidney diseases. Some topics addressed were the present and future genetic diagnosis, the use of multi-gene panels in renal or skeletal diseases, the role of animal models to better understand underlying skeletal changes, and the role of conventional radiology and surgery in the diagnosis and final treatment of bone deformities; all these without forgetting the important role of FGF23 and Klotho imbalances that result in the genetic change causing this disease. The optimization and limitations of conventional treatments currently available was also a topic addressed extensively, as well as the implications that new treatments against FGF23 could have in the future. This article is based on previously conducted studies and does not contain any new studies with human participants or animals performed by the author.

Keywords: FGF23; Klotho; Metabolic skeletal dysplasias; Renal failure; X-linked hypophosphataemic rickets

Metabolic skeletal dysplasias are primary bone diseases caused by metabolic changes which result in abnormalities in the bone structure. These metabolic changes consist mainly in
A. González-Meneses López ( $\varangle)$

Unidad de Dismorfología, Unidad de Gestión

Clínica de Pediatría, Hospital Universitario Virgen

del Rocío, Sevilla, Spain

e-mail: antonio.gonzalezmeneses.l.sspa@

juntadeandalucia.es 
phosphorus and calcium level imbalance or in the deposit of external substances which alter the bone matrix resulting in deformities, short stature and changes in other organs and systems among which the kidney is of great importance. Most of these processes are caused by inherited genetic disorders which may be dominant, recessive or linked to the $\mathrm{X}$ chromosome [1].

In the diagnosis of these entities we must take into account both the family history and the skeletal, biochemical and genetic changes that may occur. The treatment of these diseases has been primarily focused on the symptomatic correction of abnormalities in the phosphate-calcium metabolism or on the surgical correction of skeletal deformities. However, the underlying causes of most of these disorders are not yet fully understood [1]. Nonetheless, in recent years we have seen the development of new therapies that are radically changing the way some of the metabolic skeletal dysplasias are addressed. Also, the underlying biochemical and biological phenomena involved in the development and evolution of these diseases as well as their genetics and the familial implications are better understood $[1,2]$.

In November 2018, the 2nd meeting on the diagnosis and follow-up of X-linked hypophosphataemic rickets $(\mathrm{XLH})$, sponsored by Kyowa Kirin Pharmaceuticals, took place in Madrid, bringing together a good number of national experts who gave several lectures and updates on this still unknown disease.

These diseases have a genetic origin. Thus, physicians must have a deep knowledge of genetics and be updated on the ways to approach the genetic diagnosis of these medical conditions. In less than 10 years we have gone from having very basic tools such as the karyotype analysis and Sanger sequencing to massive sequencing techniques or molecular karyotypes. These new approaches have three main advantages: (1) simultaneous and much faster analysis of multiple genes, (2) lower cost for the healthcare system and (3) the care of affected patients has become easier [3]. In addition, it is well known that women who carry an X-linked disorder can in turn manifest the typical changes of the disease, to a greater or lesser extent, changing their status from mere carriers to truly affected individuals. This is especially striking in X-linked hypophosphataemia. Women with one of their $X$ chromosomes carrying a pathogenic mutation can develop a clinical skeletal deformity as well as biochemical and renal abnormalities, completely comparable to those of their affected children. This makes the traditional concept of "healthy carrier" outdated in these diseases and these women become strong candidates for diagnosis and treatment of a familial disorder that they not only carry but also endure $[4,5]$.

For XLH, measuring the actual quality of bone structure is paramount. The conventional radiological techniques such as a simple X-ray, although still important, must be complemented with nuclear magnetic resonance imaging or micro fracture techniques that can allow a much better understanding of the bone quality and structure of these patients. In addition, they allow the monitoring of treatment response and the need for corrective measures if required [5].

Short statue is the main characteristic of patients with XLH. Animal models have proved that the growth plate of weight-bearing bones becomes deformed and altered in early childhood, which results in growth deformities that are not going to be corrected except by a treatment that could be aetiological $[4,5]$. The use of growth hormone in a mouse model resulted in abnormal matrix growth causing a higher linear growth but maintaining this growth plate deformity, hence the controversial results of the growth hormone $(\mathrm{GH})$ therapy in these patients [6].

When analysing the underlying mechanisms in the pathogenesis of XLH, there are two compounds that play a crucial role in the regulation of serum phosphate-calcium homeostasis-FGF23 and Klotho. They use the kidney and skeleton as a way of excreting phosphorus and calcium as well as increasing or decreasing, depending on the needs, the absorption of phosphorus and calcium in the intestine. The dysfunction of this balance, in this case due to a genetic cause, leads to intense excretion of urinary phosphorus that greatly alters the Klotho and FGF23 balance $[7,8]$, secondarily causing the serious skeletal and renal changes that 
characterize this disease. A detailed study of the phosphate-calcium balance and Klotho-FGF23 balance is essential to understand not only these renal changes so specific to XLH but also the mechanisms involved in skeletal alterations present in chronic renal failure and the increased risk of death associated with microalbuminuria, among others. Thus, a large increase in FGF23, which occurs in both XLH and chronic renal failure, has a basic role in the maintenance of phosphaturia and therefore in the production of renal and bone disease. The control of this excess FGF23 in blood can contribute significantly to the improvement of the changes present in these diseases $[5,7,8]$.

The traditional treatment of XLH has been based mainly on the secondary correction of phosphate-calcium imbalance present in patients, by repeatedly administering phosphorus supplements as well as vitamin D analogues several times a day in an attempt to restore this imbalance. This treatment, the only one available for decades, is not without complications and failures, clinical outcomes that cannot be considered satisfactory in any way. One of the main problems we find in conventional treatment is the lack of adherence, since phosphorus must be administered orally several times a day even at night, if we want to achieve a balance that, if not optimal, is at least acceptable as a treatment outcome. These phosphorus supplements usually have a highly unpleasant taste which means that many children refuse to take them, especially when reaching adolescence. On the other hand, the combination of these phosphorus supplements with vitamin $\mathrm{D}$ analogues usually results in renal calcinosis, which too frequently leads to treatment discontinuation or limited treatment. Furthermore, clinical response in terms of growth and prevention of deformities is very variable from one patient to another even within the same family and with a similar degree of compliance, so clearly we are not facing a treatment that meets all our therapeutic expectations $[5,7,8]$.

Once skeletal deformities are established, mainly the arching of the lower limbs, there are few therapeutic options capable of reversing this deformity that do not involve surgical measures to a greater or lesser extent. These corrective surgeries can be performed not only to improve curvature but sometimes are accompanied by skeletal elongations that can also improve the individual's final size. Individualized planning of these surgeries is essential to have not only an aesthetic but also an adequate functional result and the surgical team must be realistic in terms of outcomes. The implementation of computer-assisted surgical correction techniques and computer simulations is a major advance in this surgical field, as well as the application of new osteosynthesis materials such as electromagnetic nails. However, one factor that remains critical is the experience accumulated by the centres that are able to perform these surgeries, since as a result of their complexity this experience is not available to all centres [5].

In summary, a good diagnostic approach that includes both biochemical and genetic diagnostic algorithms, not only based on calcium and phosphorus levels but also accompanied by comprehensive genetic studies, determination of Klotho and FGF23 levels, and adequate imaging techniques will allow one to accelerate the times and reliability of diagnoses that are not always easy to make. The emergence of much more specific treatments such as FGF23 antibodies in recent years seems to be able to change the paradigm of approaching high-burden diseases that have been orphaned for years, by a real improvement in their treatment and follow-up. It is clear that the emergence of an aetiological treatment for a rare disease clearly implies progress in the knowledge of its underlying biochemical factors, as well as an improvement in the care of these patients; furthermore, an improvement not only for a disease for which there is treatment but also for all those other similar diseases that share some pathophysiological mechanisms. Ultimately, this means an important advance for diseases forgotten in many cases [9].

\section{ACKNOWLEDGEMENTS}

This supplement has been funded by Kyowa Kirin. The Guest Editor would like to thank the 
participants and lecturers in the conferences for their high level of involvement and excellence and the great ease with which they transmitted their knowledge on difficult topics in such an easy manner; and Kyowa Kirin Pharmaceuticals for making this meeting possible.

Funding. Kyowa Kirin organized the scientific meeting and contributed to the financing of the publication of the opinion of the speakers presented at that meeting (Madrid, November 2018).

Medical Writing, Editorial, and Other Assistance. The author would like to thank Ana María Palma Nieto for providing translation and copyediting assistance on behalf of Springer Healthcare. Kyowa Kirin funded the writing assistance provided by Springer Healthcare Ibérica S.L.

Authorship. The named author meets the International Committee of Medical Journal Editors (ICMJE) criteria for authorship for this editorial, takes responsibility for the integrity of the work as a whole, and has given approval for this version to be published.

Disclosures. Antonio González-Meneses López declares no conflict of interest for the preparation and publication of this manuscript.

Compliance with Ethics Guidelines. This editorial is based on previously conducted studies and does not contain any new studies with human participants or animals performed by the author.

\section{REFERENCES}

1. Nikkel SM. Skeletal dysplasias: what every bone health clinician needs to know. Curr Osteoporos Rep. 2017;15(5):419-24.

2. Langeveld M, Hollak CEM. Bone health in patients with inborn errors of metabolism. Rev Endocr Metab Disord. 2018;19(1):81-92.

3. Yohe S, Thyagarajan B. Review of clinical nextgeneration sequencing. Arch Pathol Lab Med. 2017;141(11):1544-57.

4. Skrinar A, Dvorak-Ewell M, Evins A, et al. The lifelong impact of X-linked hypophosphatemia: results from a burden of disease survey. J Endocr Soc. 2019;3(7): 1321-34.

5. Haffner D, Emma F, Eastwood DM, et al. Clinical practice recommendations for the diagnosis and management of X-linked hypophosphataemia. Nat Rev Nephrol. 2019;15(7):435-55.

6. Fuente R, Gil-Peña H, Claramunt-Taberner D, et al. Marked alterations in the structure, dynamics and maturation of growth plate likely explain growth retardation and bone deformities of young Hyp mice. Bone. 2018;116:187-95.

7. Imel EA, Biggin A, Schindeler A, Munns CF. FGF23, hypophosphatemia, and emerging treatments. JBMR Plus. 2019;3(8):e10190.

8. Beck-Nielsen SS, Mughal Z, Haffner D, et al. FGF23 and its role in X-linked hypophosphatemia-related morbidity. Orphanet J Rare Dis. 2019;14(1):58.

9. Imel EA, Glorieux FH, Whyte MP, et al. Burosumab versus conventional therapy in children with $\mathrm{X}$-linked hypophosphataemia: a randomised, activecontrolled, open-label, phase 3 trial. Lancet. 2019;393(10189):2416-27. 